Classification

Physics Abstracts

$61.25 \mathrm{H}-78.20 \mathrm{~J}-82.70 \mathrm{D}$

\title{
Electric Field Light Scattering by Rod-Like Polyelectrolytes in Aqueous Suspensions
}

\author{
C. Martin, B. Weyerich, J. Biegel, R. Deike, C. Johner, R. Klein and R. Weber \\ Fakultät fur Physık, Universıtät Konstanz, 78434 Konstanz, Germany
}

(Received 18 November 1994, revised in final form 23 January 1995, accepted 25 January 1995)

\begin{abstract}
Static light scattering measurements are presented for rod-like fd-virus particles ( $L=880 \mathrm{~nm}, D=9 \mathrm{~nm}$ ) subjected to a pulsed alternating electric field in aqueous suspensions at very low ionic strength. In aqueous suspensions the dispersed fd-particles are negatively charged and surrounded by a diffuse Debye counterion cloud. In an external electric field an induced dipole originating from a deformation of the diffuse counterion cloud causes the alignment of the macromolecules. The anisotropic orientation distribution of the particles in the presence of the electric field results in a change of the angular distribution of the scattered light intensity with regard to the isotropic case. The steady-state electric field light scattering effect $\Delta I / I_{0}$ is measured as a function of the electric field strength and its frequency at a fixed scatternng angle. The determination of the anisotropy of the electric polarizability $\Delta \alpha_{e l}$ of a fd-virus particle at higher electric field strengths, above the Kerr regime, shows a decrease of $\Delta \alpha_{\mathrm{el}}$ with increasing field. This is interpreted as a destruction of the diffuse Debye cloud in high electric fields. The orientational order parameter has been found to be as large as 0.93 indicating an almost complete particle orientation along the external field at the highest fields. It is also shown that in the frequency regime below $1 \mathrm{kHz}$ electrostatically interacting rods can align perpendicular to the external electric field, whereas at higher frequencies this anomalous behaviour disappears. From the scattered intensity the form-factor and the static structure factor of interacting fd-virus particles have been determined. With increasing fields a substantial increase in the peak height of the static structure factor is found. The data is in good agreement with Monte Carlo simulations using a simple interaction model for the system The orientation of the macromolecules in the presence of an electric field is affected by the intermolecular electrostatic repulsion.
\end{abstract}

\section{Introduction}

Aqueous suspensions of fd-virus particles have been used to study phenomena like diffusion, structural ordering, orientation in external fields, phase transitions, etc. The influence of an electric field applied to a suspension of macromolecules is of considerable theoretical and practical interest. In the case of electrorheological fluids the external field induces a chain-like structure of the dispersed particles along the field lines and a liquid-solid phase transition occurs $[1,2]$. Much less is known about the change of the microstructure of suspensions of charged non-spherical macromolecules due to an orienting field [3], especially if interparticle 
correlations originating from the electrostatic repulsion between the macromolecules determine predominately the suspension properties.

We report electric field light scattering investigations on aqueous suspensions of fd-virus particles at low ionic strength and compare their results with those of theory and computer simulations. The fd-virus is a filamentous bacteriophage with a length of $L=880 \mathrm{~nm}$, a diameter of $D=9 \mathrm{~nm}$, a molecular weight of $16.4 \times 10^{6}$ Dalton and a very low polydispersity $[4,5]$. It is only slightly flexible and a good representatıve for a rod-like particle [5-7]. The particle concentrations of the examined fd-virus suspensions are below and above the overlap concentration $c^{*}$ which is defined as: $c^{*}=1$ partıcle $/$ length ${ }^{3}=0.04 \mathrm{mg} / \mathrm{ml}$. At $\mathrm{pH}=7.4$ the fd-virus has approximately 9000 ionizible groups on its surface [8] In aqueous solution this gives rise to an effective negative charge of about $400 \mathrm{e}^{-}$on each virus [9] which is surrounded by a diffuse counterion cloud characterized by the Debye-Hückel parameter $\kappa$ [10]. In highly delonized water the resulting long range Coulomb interactions force the dispersed macromolecules to form spatially ordered states even at relatively small particle concentrations, when hard core interaction is still negligible. The interparticle interaction depends on the particle concentration as well as on the ionic strength At weaker interparticle interaction this leads to a short range or liquid-like ordering of the centers of mass. For anisodiametric particles a long range translational order or/and a long range orientational order can be found at stronger interparticle interaction (liquid-crystalline phase). The interparticle electrostatic repulsion can be screened by adding salt to the suspension. The fd-suspensions studied in the present investigation exhibit elther liquid-like short range order or no order at all; therefore the partıcles are isotropically oriented without an external electrıc field.

The investigation of the electric field light scattering effect of fd-virus particles complete and extend our previous electric birefringence measurements [11-13] which have shown that an induced dipole mechanism orients the macromolecules in an electric field. The induced dipole originates predominantly from a polarization of the diffuse part of the counterion cloud with respect to the hard-core of the rod. Therefore the extension of the counterion cloud strongly contributes to both the interaction of a single fd-virus particle with an external electric field and the interparticle interaction of the rods. At very low ionic strengths the extension of the counterion cloud can be several hundred nanometers causing a strong overlap of the diffuse counterion clouds for particle concentrations above $c^{*}$. The steady-state value of the relative change of the scattered light intensity $\Delta I / I_{0}$ in an electric field yields information about the orientation of the suspended particles $[14,15]$. For various particle concentrations we measured the electric field light scatterıng effect $\Delta I / I_{0}$ at a fixed scattering angle as a function of the electric field strength and the field frequency.

In order to investigate the influence of an anisotropic orientation distribution on the configuration of the rods we examined the scattered intensity (steady-state) in an external electric field $I(\mathbf{q}, \mathbf{E})$ as a function of the scattering vector $\mathbf{q}$. At sufficiently high ionic strength the Coulomb interaction is screened and no ordering is observed. In this case the scattered intensity corresponds to the one-particle form-factor $P(\mathbf{q})$. The evaluation of the particle form-factor in an electric field $P(\mathbf{q}, \mathbf{E})$ is based on a theoretical treatment for Rayleigh-Gans-Debye scattering (RGD) by Stoylov [16] and Stoimenova [17]. The results derived by Stoimenova for non-interacting particles of cylindrical symmetry are applied taking the limit of infinitely thin rods. In the Kerr regime the anisotropy of the electric polarizability $\Delta \alpha_{\mathrm{el}}$ is known and the numerical results for $P\left(\mathbf{q}, \mathbf{E}, \Delta \alpha_{\mathbf{e l}}\right)$ can be compared directly to the experimental data. At higher electric fields $\Delta \alpha_{\mathrm{el}}$ becomes electric field strength dependent, and $\Delta \alpha_{\mathrm{el}}(E)$ can be determined from a comparison of experimental data and numerical calculations. In suspensions of very low ionic strength a liquid-like structure of the particles with a significant first intensity maximum of the elastically scattered light is observed. To obtain the static structure factor in an electric 
field $S(\mathbf{q}, \mathbf{E})$ the measured intensity $I(\mathbf{q}, \mathbf{E})$ is divided by the calculated form-factor $P(\mathbf{q}, \mathbf{E})$. For interacting systems the form-factor $P(\mathbf{q}, \mathbf{E})$ fits the data at large scattering angles where $S(\mathbf{q}) \approx 1$.

Furthermore, the electric field light scattering results are reproduced by Monte-Carlo (MC) simulations. The $\mathrm{MC}$ simulations are based on a model potential describing a screened Coulomb interaction between the particles (DLVO), as well as the coupling to an external electric field. For isotropic suspensions of charged rod-like polyelectrolytes, MC simulations using the DLVO potential have been applied successfully to describe their scattering properties $[18,19]$. Simulation results give also insight into details of the microstructure of the suspension which are not accessible by light scattering experiments.

\section{Theoretical Considerations}

2.1. General Description. - In a static light scattering experiment (SLS) the time averaged scattered intensity $I(\mathbf{q})$ of a disperse system of $N$ monodisperse interacting particles is measured. The intensity of the scattered light within the Rayleigh-Gans-Debye approximation (RGD) can be expressed by the one-particle form-factor $P(\mathbf{q})$ and the static structure factor $S(\mathbf{q})[20]:$

$$
I(\mathbf{q}) \propto N P(\mathbf{q}) S(\mathbf{q}),
$$

where the magnitude of the scattering vector $q$ is

$$
q:=|\mathbf{q}|=\frac{4 \pi}{\lambda} \sin \left(\frac{\vartheta}{2}\right) .
$$

Here, $\lambda$ is the wavelength of the incident light in the suspension and $\vartheta$ denotes the angle of observation or scattering angle. For the RGD approximation [21] to be satisfied the condition $(4 \pi L / \lambda)\left|\left(n_{\mathrm{P}} / n_{\mathrm{S}}\right)-1\right| \ll 1$ imposes a restriction on the length $L$ of the particles and its relative refractive index $n_{\mathrm{P}} / n_{\mathrm{S}}$ (particle - solvent). Recently the validity of the RGD approximation for rigid rods of a length of the order of the incident wavelength and a relative refractive index $n_{\mathrm{P}} / n_{\mathrm{S}}<1.2$ has been verified [22] by comparing to numerical calculations based on the Coupled Dipole Method. It is therefore assumed that the RGD approximation can be used in the present study. The application of an electric field to suspensions of electrically anisotropic macromolecules results in a change of the angular distribution of the scattered light. The relative change of the light intensity in an external field scattered by anisodiametric particles is defined by $[23,24]$ :

$$
\frac{\Delta I}{I_{0}}=\frac{I(\mathbf{q}, \mathbf{E})-I_{0}(\mathbf{q})}{I_{0}(\mathbf{q})}
$$

$I(\mathbf{q}, \mathbf{E})$ denotes the intensity of the scattered light when the particles are subjected to an electric field and $I_{0}(\mathbf{q})$ is the scattered intensity of isotropically oriented rods in the absence of an electric field.

The fd-virus particles are modeled as monodisperse rigid rods of length $L[5,6]$ and vanishing diameter. As far as light scattering is concerned the very small optical anisotropy of the fd-virus particles can be neglected. The suspension should be sufficiently diluted and therefore free of multiple scattering effects. The incident light is plane polarized. The intensity of light $\imath(\mathbf{q}, \mathbf{u})$ scattered by an infinitely thin cylinder within the RGD approximation is given by $[21,25]$ : 


$$
\imath(\mathbf{q}, \mathbf{u})=C\left|\jmath_{0}\left(\frac{L}{2} \mathbf{q} \cdot \mathbf{u}\right)\right|^{2}
$$

where $C$ is a factor independent of particle geometry and $j_{0}$ is the spherical Bessel function of order zero. The unit vector $u$ denotes the orientation of a rod.

2.2. Particle Form-FaCtor. - If the Coulomb interaction between the particles is screened $(S(q) \equiv 1)$ the scattered intensities of the macromolecules inside the scattering volume are only due to their intramolecular interferences. In general the orientation distribution of the particles caused by an electric field is time dependent. After a rise process, when the electric field has been applied long enough, the intensity change of the scattered light $\Delta I$ reaches a constant value. In that steady-state regime the orientation distribution function $f(\mathbf{u})$ of the macromolecules is independent of time and the one-particle form-factor can be calculated by the orientational ensemble average:

$$
P(\mathbf{q})=\frac{1}{C}\langle\imath(\mathbf{q}, \mathbf{u})\rangle_{\text {orient }}=\frac{1}{C} \int \mathrm{d} \mathbf{u} \imath(\mathbf{q}, \mathbf{u}) f(\mathbf{u}),
$$

where $\int \mathrm{d} \mathbf{u} \ldots=\int_{0}^{2 \pi} \int_{0}^{\pi} \mathrm{d} \Phi \mathrm{d} \theta \sin \theta \ldots$ denotes the integration over the solid angle.

(i) isotropic particle orientation $(E=0)$ :

For an optically isotropic suspension the orientation distrubution function is constant: $f(\mathbf{u})=$ $1 / 4 \pi$. The isotropic one-particle form-factor $P_{0}(q)$ is calculated as [20]:

$$
P_{0}(q)=\frac{2}{q L} \int_{0}^{q L} \frac{\sin z}{z} \mathrm{~d} z-\left(\frac{2}{q L} \sin \left(\frac{q L}{2}\right)\right)^{2} .
$$

(ii) anisotropic particle orientation $(E \neq 0)$ :

The external field causes an anisotropic orientation distribution of the rods and the suspension becomes optically anisotropic (birefringent). In the steady-state regime the orientation distribution function $f(\mathbf{u})$ is described by a Boltzmann distribution. Assuming the field direction parallel to the unit vector $\mathbf{e}_{z}$ the orientation distribution function depends only on the polar angle $\theta$ [14]:

$$
f(\theta)=\frac{\mathrm{e}^{-U_{\text {orient }} / k_{\mathrm{B}} T}}{\int \mathrm{d} \mathbf{u} \mathrm{e}^{-U_{\text {orient }} / k_{\mathrm{B}} T}}=\frac{\mathrm{e}^{-U_{\text {orient }} / k_{\mathrm{B}} T}}{2 \pi \int_{0}^{\pi} \mathrm{d} \theta \sin \theta \mathrm{e}^{-U_{\text {orient }} / k_{\mathrm{B}} T}},
$$

where $U_{\text {orient }}$ is the potential energy of a single rod in an external field and $\theta$ is the angle between the symmetry axis of a rod and the external electric field. The alignment of the fdvirus particles is caused by an induced dipole mechanism which is determined by the electric field and the symmetric polarizability tensor consisting of the electric polarizabilities parallel $\alpha_{\mathrm{el}}^{\| l}$ and perpendicular $\alpha_{\mathrm{el}}^{\perp}$ to the rod. The potential energy $U_{\text {orient }}$ of a fd-virus particle in a homogeneous electric field relative to $k_{\mathrm{B}} T$ can be written as $[26,27]$ :

$$
\frac{U_{\text {orient }}}{k_{\mathrm{B}} T}=-\frac{\Delta \alpha_{\mathrm{el}} E^{2}}{2 k_{\mathrm{B}} T} \cos ^{2} \theta=:-\gamma \cos ^{2} \theta
$$

Here, $\Delta \alpha_{\mathrm{el}}:=\alpha_{\mathrm{el}}^{\|}-\alpha_{\mathrm{el}}^{1}$ denotes the electric anisotropy (polarizability) of a rod. The parameter $\gamma$ reflects the ratio of the orientation energy $U_{\text {orient }}$ and the thermal energy $k_{\mathrm{B}} T$. 
In our experimental set-up the electric field direction is fixed perpendicular to the plane of observation, i.e., $\mathbf{q} \perp \mathbf{E}$. Since the system is axially symmetric with respect to the external field, the scattering intensity depends only on the absolute value of the scattering vector $q$. For complete particle orientation along the field the orientation distribution function is simply a delta function: $f(\mathbf{u})=\delta\left(\mathbf{u}-\mathbf{e}_{z}\right)$, and the particle form-factor for infinitely high field strength becomes a constant: $P(q, \gamma \rightarrow \infty)=1$, so that $\Delta I(q, \gamma \rightarrow \infty) / I_{0}=\left(1 / P_{0}(q)\right)-1$. In order to obtain the relative change of the scattered light intensity $\Delta I / I_{0}$ for arbitrary electric field strengths Stoimenova [17] has used an expression similar to equation (5) and carried out a Taylor expansion for the integrand. For infinitely thin rods the steady-state value of the particle form-factor in an external field $P(q, \gamma)$ can be expressed by:

$$
\begin{gathered}
P(q, \gamma)=\frac{16 \pi}{T_{n}(\gamma)}\left\{\sum_{p=0}^{\infty} \frac{\gamma^{2 p}(4 p-1) ! !}{(2 p) !(4 p) ! !} \sum_{m=0}^{\infty} \xi_{p, m}(q L)^{2 m}\left[1+\zeta_{p, m} \gamma\right]\right\}-1 \\
\xi_{p, m}=\frac{(-1)^{m}(2 m-1) ! !(4 p) ! !}{2(2 m+2) !(2 m+4 p+1) ! !} \\
\xi_{p, m}=\frac{(4 p+1)}{(2 p+1)(2 m+4 p+3)}
\end{gathered}
$$

The normalizing factor $T_{n}(\gamma)=\int \mathrm{du} \mathrm{e}^{-U_{\text {orient }} / k_{\mathrm{B}} T}=2 \pi \int_{0}^{\pi} \mathrm{d} \theta \sin \theta \exp \left(\gamma \cos ^{2} \theta\right)$ was tabulated by Dawson [28]; it can easily be calculated numerically.

For small electric field strengths at low degree of particle orientation equation (9) reduces to results derived by Wippler [24]. The electro optic effect (EOE) is then directly related to the electric polarizabilities of a single particle [14]. The relative change of the scattered intensity $\Delta I / I_{0}$ in the Kerr regime is calculated as:

$$
\frac{\Delta I}{I_{0}}\left(q L, E, \Delta a_{\mathrm{el}}\right)=\left\{\frac{1}{6}+\frac{2}{P_{0}(q L)}\left[\frac{\sin (q L)}{(q L)^{3}}-\frac{1}{(q L)^{2}}\right]\right\} \frac{\Delta \alpha_{\mathrm{el}} E^{2}}{2 k_{\mathrm{B}} T}
$$

The prefactor is determined by the magnitude of the scattering vector $q$ and the length of a rod.

In Figure 1 the calculated relative change of the scattered light intensity $\Delta I(q, \gamma) / I_{0}(q)$ and the form-factor $P(q, \gamma)$ are plotted for various $\gamma$-values as a function of $q L$ to exhibit the deviations from the case of isotropic particle orientation. If the particles tend to orient perpendicular to the electric field $\left(\Delta \alpha_{\text {el }}<0\right)$, the form-factor is reduced with respect to the isotropic case. For particle alignment parallel to the external field $\left(\Delta \alpha_{\text {el }}>0\right)$ the scattered light intensity is enhanced. For finite $\gamma$ Figure 1 clearly shows that the deviations of $\Delta I(\gamma) / I_{0}$ from the saturation curve $\Delta I(\gamma \rightarrow \infty) / I_{0}$ increase with $q$ due to the form-factor. Even for highly aligned particles, e.g., $\gamma \approx 20 \rightarrow \Phi(\gamma) \approx 0.92$ (see Section 2.3 ), there are still significant deviations from the limit of totally aligned rods $\left(\mathbf{u} \| \mathbf{e}_{z}\right)$ at high $q$-values.

2.3. Orientational Order Parameter. - In this section we introduce an order parameter, which indicates the degree of particle orientation in a suspension of rod-like particles subjected to an external field. The order parameter or orientation function is related to the equations known from birefringence and electric field light scattering for small particles [14,27], wherein the measured signal $\left(\Delta n\right.$ or $\left.\Delta I / I_{0}\right)$ is simply given by the product of the order parameter and a saturation value for complete particle alignment. Since in our experimental setup the field direction is parallel to the unit vector $\mathbf{e}_{z}$, the orientational order parameter can be defined as [26]. 


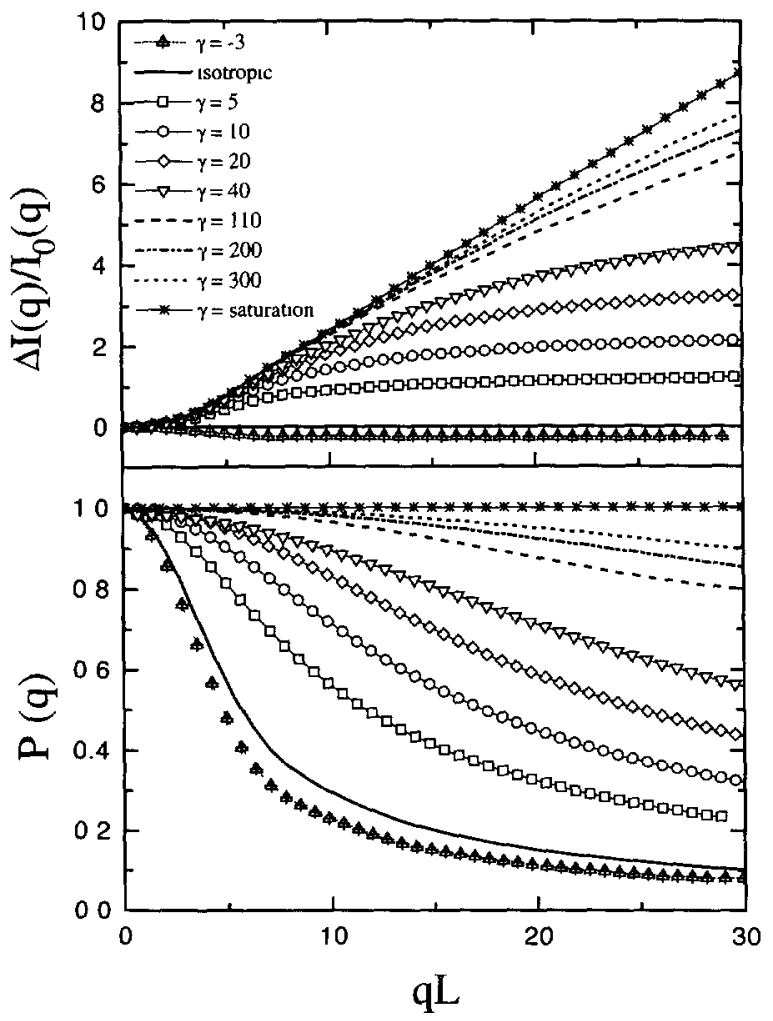

Fig. 1. - Numerical calculations according to equation (9) of the relative change of the scattered light $\Delta I(q, \gamma) / I_{0}(q)$ and the form-factor $P(q, \gamma)$. The solid line corresponds to the isotropic case $(\gamma=0)$ in the absence of an external electric field.

$$
\Phi(\gamma)=2 \pi \int_{0}^{\pi} \mathrm{d} \theta \sin \theta\left[\frac{3 \cos ^{2} \theta-1}{2}\right] f(\theta, \gamma),
$$

where $f(\theta, \gamma)$ is obtained from equations (7) and (8). The orientational order parameter solely depends on $\gamma$. For small values of $\gamma$ the order parameter increases linearly and reaches a saturation value at high field strength. The saturation value for $\Delta \alpha_{\text {el }}>0$ is $\Phi(\gamma \rightarrow \infty)=1$. Complete particle alignment perpendicular to the field lines would lead to a saturation value of $\Phi(\gamma \rightarrow-\infty)=-0.5$. The orientational order parameter $\Phi(\gamma)$ for $\Delta \alpha_{\mathrm{el}}>0$ is plotted in Figure 2.

2.4. Static Structure Factor. - In the case of interacting macromolecules the interparticle correlations give rise to intermolecular interferences. The interparticle correlations are described by the pair distribution function $g\left(\mathbf{R}, \mathbf{u}_{1}, \mathbf{u}_{2}\right)$, where $\mathbf{R}$ is the vector connecting the centers of mass of the molecules and $\mathbf{u}_{1}$ and $\mathbf{u}_{2}$ denote the directions of their symmetry axis. In the case of an isotropic suspension this quantity can be expanded in rotational invariants [29], the expansion coefficients are representing center-center and angular correlations [30]. For totally aligned rods $\left(\mathbf{u}_{1}=\mathbf{u}_{2}=\mathbf{e}_{z}\right.$ ) the expression for the pair distribution function is simplified to $g(\mathbf{R}) \equiv g(R, \theta)$ where $\theta$ is the angle between the center-center distance vector $\mathbf{R}$ and the direction of the alignment [31]. 


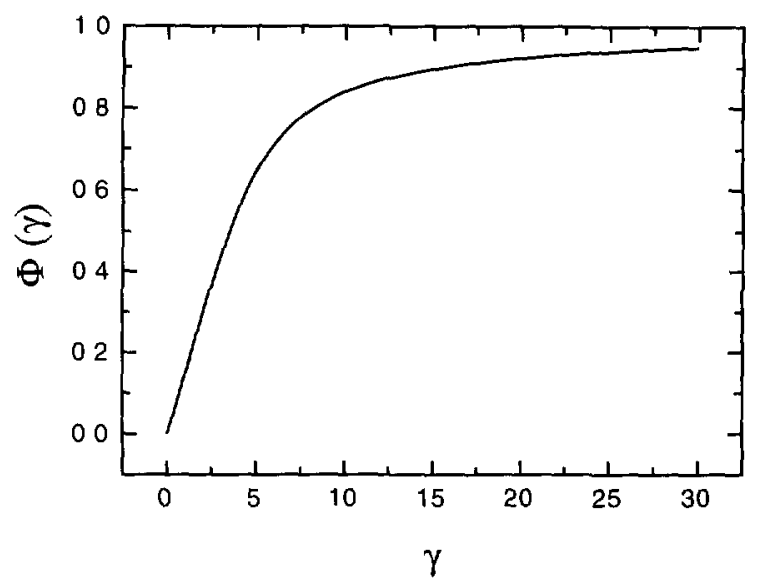

Fig 2 - The theoretical orientational order parameter $\Phi(\gamma)$ accordıng to equation (11).

The contribution of the intermolecular correlations to the scattered light intensity is given by the static structure factor $S(\mathbf{q})[18]$.

$$
S(\mathbf{q})=1+\frac{1}{N P(\mathbf{q})}\left\langle\sum_{\imath \neq j}^{N} \mathrm{e}^{\imath \mathbf{q}\left(\mathbf{R}_{\imath}-\mathbf{R}_{\jmath}\right)} j_{0}\left(\frac{L}{2} \mathbf{q} \cdot \mathbf{u}_{\imath}\right) \jmath_{0}\left(\frac{L}{2} \mathbf{q} \cdot \mathbf{u}_{\jmath}\right)\right\rangle
$$

The bracket $\langle\ldots\rangle$ denotes the canonical ensemble average. Provided that the scattering vector $\mathbf{q}$ is perpendicular to the orienting field, the static structure factor depends only on the scalar value of $\mathbf{q}$. Like the pair distribution function, $S(\mathbf{q})$ is determined by the positions of the centers of mass $R_{\imath}$ as well as by the orientations of the interacting rods. The static structure factor of strongly interacting rods has a pronounced peak. Since an analytical expression for $S(q, E=0)$ is avallable only for weakly interactıng rods [32,33], results for this quantity must be obtained from Computer Simulations.

\section{Monte-Carlo Simulation}

For low and intermediate values of the scattering vector $\mathbf{q}$, interparticle correlations described by the static structure factor $S(\mathbf{q})$ contribute substantially to the scattered light intensity. In order to calculate $S(\mathbf{q})$ we assume an interaction potential that describes the electrostatic repulsion between the macromolecules as well as the coupling to an external electric field. Using this potential we can evaluate the static structure factor by means of Monte-Carlo simulations.

As in previous studies of isotropic suspensıons of rod-like macromolecules [18] the interparticle interaction is modeled as a repulsive DLVO potential between $n$ segments of one rod with $n$ segments of another rod. The pair interaction between rods with orientation $\mathbf{u}_{1}, \mathbf{u}_{2}$ and a center of mass distance $\mathbf{R}$ is given by:

$$
U_{\operatorname{DLVO}}\left(\mathbf{R}, \mathbf{u}_{1}, \mathbf{u}_{2}\right)=\sum_{\alpha, \beta=1}^{n}\left(\frac{Q}{n}\right)^{2} \frac{\mathrm{e}^{-\kappa\left(r_{\alpha \beta}-2 a\right)}}{4 \pi \varepsilon_{0} \varepsilon_{\mathrm{r}}(1+\kappa a)^{2} r_{\alpha \beta}}
$$

Here, $Q$ is the total charge per rod, $n$ the number of segments, $\varepsilon_{0}$ the permittivity of vacuum, $\varepsilon_{\mathrm{r}}$ the relative permittivity, $a$ the hard core radius of the rod and $r_{\alpha \beta}$ the distance between segment $\alpha$ on $\operatorname{rod} 1$ and segment $\beta$ on $\operatorname{rod} 2$. In this paper we use 3 segments with a distance 
of $L / \sqrt{8}$ between segments on a rod. The quadrupolar moment of this charge distribution is equal to a homogeneously charged rod with length $L$. A cylindrical hard core of length $L$ and radius $a$ has been included in the $\mathrm{MC}$ simulations to prevent configurations with overlapping rods. The Debye-Hückel screening length $\kappa^{-1}$ describes the extension of the diffuse counterion cloud and is given by $[9,10]$ :

$$
\begin{gathered}
\kappa^{2}=\frac{e_{0}^{2}}{\varepsilon_{0} \varepsilon_{\mathrm{r}} k_{\mathrm{B}} T} I S \\
I S:=c_{\mathrm{p}} Q+\sum_{\imath} c_{2} z_{\imath}^{2},
\end{gathered}
$$

where $e_{0}$ is the elementary charge, $c_{\mathrm{p}}$ the particle concentration of the macromolecules, $z_{2}$ and $c_{2}$ are the charge number and the concentration of additional salt ions of the species $\imath$. The ionic strength $I S$ is proportional to $\kappa^{2}$ and is determined by the concentration of all microions including the $\mathrm{H}^{+}$counterions dissociated from the macromolecules and additional added salt ions.

This rather simple interaction model for charged rods has recently been justified by the results obtained by Löwen [34,35], who compared a 3-segment DLVO potential with an $a b$

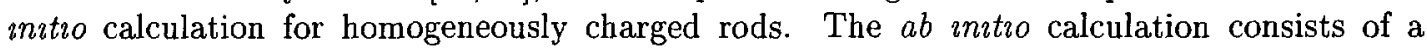
combination of molecular dynamics for the rods and classical density functional theory for the counterions. The results of both approaches coincide satisfactorily, if the value for the charge of a rod in the DLVO potential is chosen as an effective charge, which is in general lower than the real charge. In our case we consider the effective charge as a parameter which is determined by fitting the MC results for $S(q)$ to the light scattering data for an optically isotropic suspension without an external field.

The coupling to an external field has been taken into account by using the orientation dependent one-particle potential $U_{\text {orıent }}$ of equation (8). The values of $\gamma$ were taken from the experimental results. Effects beyond this mean field type description like dipole-dipole interaction between the rods have been neglected.

The MC simulations were performed applying the usual Metropolis algorithm. Each MC step consists of a random displacement of the center of mass as well as the orientation of a rod. The system size has been 256 rods, test runs with a smaller system showed no significant difference. We used a value of $Q=420 e$ for the charge per rod. After thermalization 6000-12000 configurations were used to calculate orientation distribution functions, pair correlations and the static structure factors $S(q)$. The latter was evaluated directly by performing the ensemble average given by equation (12).

\section{Experimental Section}

4.1. Materials. - A stock solution $(9.3 \mathrm{mg} / \mathrm{ml})$ of fd-virus particles was prepared with the help of Prof. Rashed (University of Konstanz) following a method of Marvin et al. [36]. Escherna coli bacteria were infected with the fd-virus. After $8 \mathrm{~h}$ at $37^{\circ} \mathrm{C}$ the fd-virus bred rapidly by a factor 1000 . The reproduction was stopped and the virus was separated from the bacteria by several steps of precipitation and centrifugation. Finally, the fd-virus suspension was ultracentrifuged in a $\mathrm{CsCl}$ gradient and dialyzed against deionized water to obtain a pure stock solution. The samples were prepared by diluting the stock solution with highly purified water $(R=18 \mathrm{M} \Omega)$. The actual particle concentrations of the samples were determined by their UV absorption at $\lambda=269 \mathrm{~nm}$ (extinction coefficient $\varepsilon=3.84 \mathrm{~cm}^{2} / \mathrm{mg}$ ), using a Beckmann spectrometer (DU-64, Darmstadt, Germany). 
4.2. Light Scattering Apparatus. - For the static light scattering investigations a light scattering apparatus (ALV, Langen, Germany) was used consisting of a computer controlled goniometer table with focussing and detector optics, a power stabilized $3 \mathrm{~W}$ argon laser (Spectra Physics), a digital photon counter, and a temperature control which stabilizes the temperature of the sample at $T=21 \pm 1^{\circ} \mathrm{C}$. A light-scattering cell was constructed with circular platinum electrodes (radius $=7 \mathrm{~mm}$; electrode distance $=1-1.5 \mathrm{~mm}$ ) providing a homogenous electric field in the scattering volume. The electric field is perpendicular to the observation plane. The cell is coupled to a tube system described in Section 4.3. The electric pulse sequences (square wave) are generated by a function generator and amplified by two wideband KrohnHite amplifiers (Model 7500, Avon, USA). At a electrode distance of $1 \mathrm{~mm}$ a maximum electric field strength of $4 \times 10^{5} \mathrm{~V} / \mathrm{m}$ can be achieved. The length of a pulse sequence (pulse frequency: $10 \leq f \leq 400 \mathrm{kHz}$ ) was chosen long enough to attain a steady-state value of the measured intensity and ranged between $100-500 \mathrm{msec}$ depending on the particle concentration and the resulting interparticle interaction [11,13]. Measurements, field generation and data acquisition are controlled by a personal computer. Intensity data are corrected by the dark-rate of the photomultplier and by the angle dependence of the scattering volume. The magnitude of the scattering vector ranges from $2.4 \times 10^{-2}$ to $3.3 \times 10^{-2} \mathrm{~nm}^{-1}$ at a laser wavelength of $\lambda=488 \mathrm{~nm}$ in aqueous suspensions with a refractıve index of $n_{\mathrm{s}}=1.33$. The scattered light intensity is determined from the mean value of 10-100 single measurements consisting of a $50 \mathrm{msec}$ integration of the detected counts. The experimental error is estimated to approximately 3-5\% and all measurements could be reproduced. The application of pulsed alternating electric fields reduces the difficulties from the associated conductivity (heating, electrode polarization, etc.) and electrophoretic effects $[15,37]$ resulting in a better reproducibility of the data than in continuoues electric fields.

4.3. Tube System. - - In a closed circuit including either the electric birefringence or the electric field light scattering cell the samples were deionized by pumping them with a tube pump through mixed-bed-ion-exchange-resin (MB3, Serva Diagnostics, Heidelberg, Germany) until the minimal ionic strength was achieved $[38,39]$. The ionic strength depends on the particle concentration. At minimal ionic strength the suspension can be considered free of small ions other than $\mathrm{H}^{+}$and $\mathrm{OH}^{-}$. The conductivity of the suspension was measured with a Knick conductometer (Knick, Berlin, Germany) in a conductivity cell (LA01, WTW, Weilheim, Germany) coupled to the tube system.

\section{Results and Discussion}

Experimental results obtained by the method of electric field light scattering and results of MC simulations for suspensions of charged rod-like macromolecules are discussed. We focus our interest on the steady-state value of the electro-optic effect (EOE), the relative change of the scattered light intensity $\Delta I / I_{0}$, of aqueous fd-virus suspensions subjected to square wave electric pulses. The stationary value is attained for pulse sequences between 100 and $500 \mathrm{~ms}$ depending on the particle concentration and the ionic strength of the suspension [11]. Different aspects of these investigations are discussed in the following sections.

5.1. Electric Field Strength Dependence. - Figure 3 shows the field strength dependence of $\Delta I / I_{0}$ for a sample with a fd-virus concentration of $0.6 c^{*}$ at various ionic strengths. The field frequency is $10 \mathrm{kHz}$ and the scattering angle is fixed to $140^{\circ}(S(q) \approx 1)$. The ionic strength is varied from approximately $10^{-6}$ to $10^{-4}$ molar by adding small amounts of $\mathrm{NaCl}$ into the pump circuit. The conductivity $(\sigma)$ of the sample, which is directly related to the 


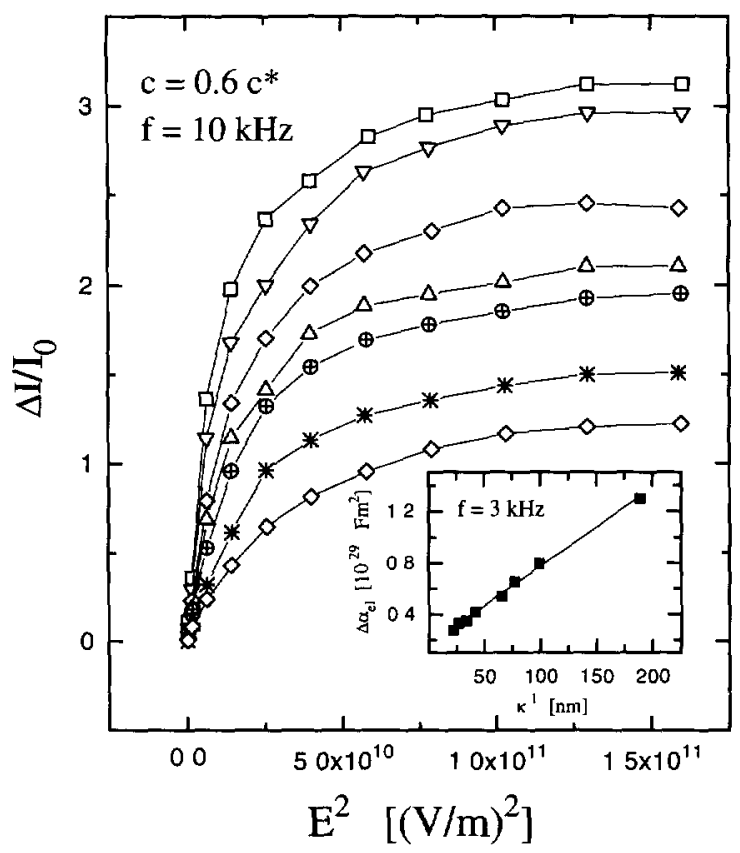

Fig. 3. - The field strength dependent $\Delta I / I_{0}$ of a fd-virus suspension with a concentration of $0.6 c^{*}$ for various conductivities (starting from the top): $0.39 \mu \mathrm{S} / \mathrm{cm}, 0.75 \mu \mathrm{S} / \mathrm{cm}, 155 \mu \mathrm{S} / \mathrm{cm}, 2.2 \mu \mathrm{S} / \mathrm{cm}$, $2.9 \mu \mathrm{S} / \mathrm{cm}, 6.4 \mu \mathrm{S} / \mathrm{cm}, 9.9 \mu \mathrm{S} / \mathrm{cm}$. The inset shows the electric anısotropy $\Delta \alpha_{\mathrm{el}}$ of a fd-virus particle as a function of the Debye-Hückel screening length $\kappa^{-1}$ (Eq. (14)). The scattering angle is $140^{\circ}$ and the frequency of the external electric field is fixed to $10 \mathrm{kHz}$ (inset $f=3 \mathrm{kHz}$ ).

Ionic strength $I S[9,39]$, is measured via a conductometer. For all ionic strengths $\Delta I\left(E^{2}\right) / I_{0}$ shows Kerr behaviour at moderate electric field strengths of $E \leq 3 \times 10^{4} \mathrm{~V} / \mathrm{m}$ and the EOE starts to saturate at electric field strengths of $E \geq 2 \times 10^{5} \mathrm{~V} / \mathrm{m}$. The saturation value of $\Delta I / I_{0}$ increases with decreasing ionic strength. The maximum $\Delta I / I_{0}$ value for the sample at minimal ionic strength is far below the theoretical value of $\Delta I / I_{0}$ for totally aligned rods $\left(\mathbf{u} \| \mathbf{e}_{z}\right.$ ) plotted in Figure 1. Provided that the particles are not destroyed in high electric fields this is caused by an incomplete particle alignment $\left(\phi\left(\gamma_{\max } \approx 20\right) \approx 0.92\right)$ and the enormous length of the fd-virus particles respectively their form-factor $P(q)$ as stated in Section 2.2. That the macromolecules stay physically intact is confirmed by the proper structure of the samples after the application of electric fields. The mechanism behind the incomplete particle orientation in high electric fields will be discussed later on.

From our previous electric birefringence investigations [13] we concluded that the orientation of the fd-virus particles at low ionic strengths is predominantly determined by the diffuse part of the counterion cloud. In this paper we confirm these results by means of electric field light scattering measurements in alternating electric fields. In the Kerr regime the electric anisotropy of a macromolecule $\Delta \alpha_{\mathrm{el}}$ can be determined by equation (10) where only the length, temperature and field strength are necessary input parameters. In contrast, the determination of $\Delta \alpha_{\mathrm{el}}$ in an electric birefringence experiment requires the additional information about the optical anisotropy of the macromolecule, which is related to the saturation value of the refractive index difference $\Delta n_{\text {sat }}$ for totally aligned particles. Previously we have measured this saturation value for fd-virus suspensions in a magnetic field [40]. Taking into account the 
measured saturation value of $\Delta n_{\text {sat }} / \mathrm{c}=3 \times 10^{-5} \mathrm{ml} / \mathrm{mg}$ the resulting electric anisotropies determined with electric birefringence agree well with the electric field light scattering results. The inset of Figure 3 shows the dependence of the electric anisotropy $\Delta \alpha_{\mathrm{el}}$ on the ionic strength $\left(f=3 \mathrm{kHz} ; \vartheta=140^{\circ}\right)$. For low ionic strengths the electric anisotropy $\Delta \alpha_{\mathrm{el}}$ increases linearly with the Debye-Hückel screening length $\kappa^{-1}$, i.e., the extension of the diffuse counterion cloud. A similar behaviour was found for charged rod-like DNA fragments $[41,42]$, and the dominant influence of the diffuse part of the counterion cloud on the particle orientation was concluded by the authors. Theoretical considerations by Rau and Charney concerning DNA particles support this interpretation $[43,44]$. In agreement with experimental results they predict the approximate proportionality of the polarization of the diffuse counterion cloud to $\kappa^{-1}$.

5.2. Dispersion of $\Delta I / I_{0}$. - In Figure 4 the frequency dependence of $\Delta I / I_{0}$ in the Kerr regime $\left(E=2.7 \times 10^{4} \mathrm{~V} / \mathrm{m}\right)$ for various particle concentrations at minimal ionic strength is plotted $\left(\vartheta=140^{\circ}, T=21^{\circ} \mathrm{C}\right)$. The EOE decreases with increasing particle concentration and shows a broad maximum at about $3 \mathrm{kHz}$ for all samples. In the low frequency regime $(f \leq 500 \mathrm{~Hz})$ the electric field light scattering effect becomes negative for higher particle concentrations. This anomalous behaviour is found for many strongly interacting systems $[13,45-48]$. At high frequencies above $400 \mathrm{kHz}$ the EOE vanishes.

Our investigations exhibit the predominant influence of the diffuse counterion cloud on the orientation of suspended fd-virus particles in an external electric field. In the Kerr regime the extension of the counterion cloud characterized by $\kappa^{-1}$ (Eq. (14)) determines the electric anisotropy $\Delta \alpha_{\text {el }}$. Since the ionic strength of a suspension strongly depends on the concentration of the suspended macromolecules, $\Delta I / I_{0}$ decreases with increasing particle concentration.

The dispersion of $\Delta I / I_{0}$ agrees rather well with our previous birefringence measurements [13] performed in sinusoidal electric fields where the maximum is found in the same frequency range. At a constant electric field strength the maximum of $\Delta I / I_{0}$ in the dispersion (Fig. 4) can only

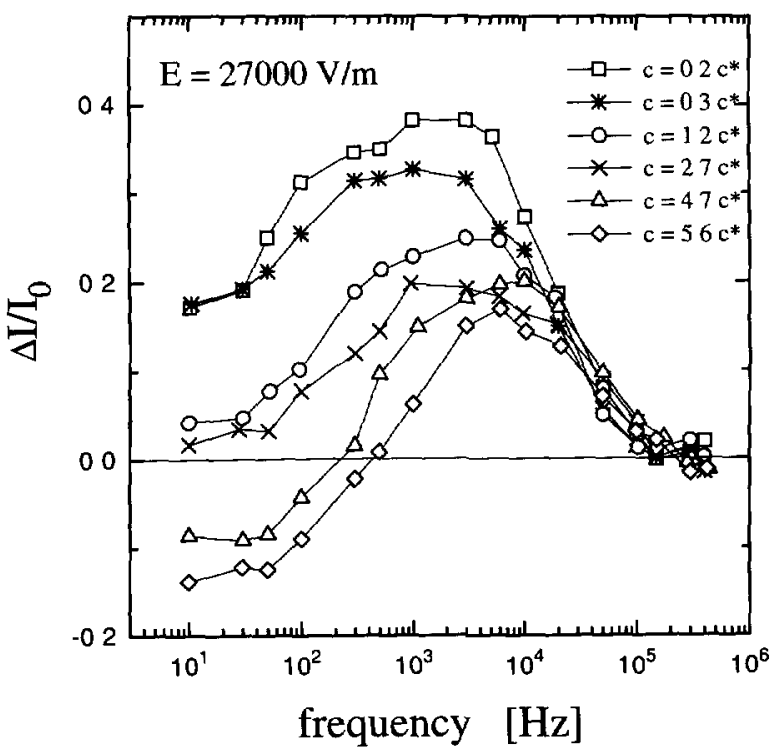

Fig. 4. - The frequency dependence of $\Delta I / I_{0}$ of fd-virus suspensions at minimal conductivities for an electric field amplitude of $27000 \mathrm{~V} / \mathrm{m}$. The scattering angle is fixed to $140^{\circ}$. 
be explained by a maximum in the dispersion characteristic of $\Delta \alpha_{\mathrm{el}}=\alpha_{\mathrm{el}}^{\|}-\alpha_{\mathrm{el}}^{\frac{1}{}}$ (see Eq. (10)). In the case of extended counterion clouds Fixmann and Jagannathan [49] showed that $\alpha_{\mathrm{el}}^{\perp}$ takes a considerable magnitude with respect to $\alpha_{\mathrm{el}}^{\|}$Assuming $\alpha_{\mathrm{el}}^{\perp}$ not far from $\alpha_{\mathrm{el}}^{\|}$a positive maximum occurs if $\alpha_{\mathrm{el}}^{\frac{1}{}}(f)$ decreases somewhat faster than $\alpha_{\mathrm{el}}^{\|}(f)$ with increasing frequency. The problem of the faster decay of $\alpha_{\mathrm{el}}^{\perp}(f)$ has been discussed in reference [13], but a definite explanation cannot be given yet. At frequencies in the range of $2 \times 10^{5}$ to $4 \times 10^{5} \mathrm{~Hz}$ the EOE vanishes and the alignment of the rod-like macromolecules in an external field disappears. At minimum ionic strength the diffuse counterion cloud is mainly build up by $\mathrm{H}^{+}$counterions dissociated from the acidic surface groups of the virus. The orientation of the macromolecules in an external electric field is caused by the polarization of the counterion cloud with respect to the negatively charged macromolecule. Therefore the relaxation time $\tau$ of such an induced dipole is correlated to the translation diffusion constant $D$ of the counterions and can be estimated by an expression given by Oosawa $[13,50]$ :

$$
\tau=\frac{L^{2}}{(2 \pi)^{2} D}
$$

To estimate $D$ we use the value of free $\mathrm{H}^{+}$ions at $20^{\circ} \mathrm{C}$ which is $8.5 \times 10^{-9} \mathrm{~m}^{2} \mathrm{~s}^{-1}$ [51] which leads to a high frequency limit of about $400 \mathrm{kHz}$. In this frequency range the induced dipole can no longer follow the stimulating electric field and the rods are no longer oriented.

Above the Kerr regime the frequency behaviour of $\Delta I / I_{0}$ deviates from that at low degree of particle orientation plotted in Figure 4. Increasing the field strength shifts the dispersion maximum from $3 \mathrm{kHz}$ to approximately $10-20 \mathrm{kHz}$. The shift of the dispersion maximum with increasing field strength could be understood in the following way: the induced dipole moment of a rod is related to the difference of the polarizability parallel and perpendicular to a rod axis. Increasing the field amplitude causes a stronger particle alignment along the field lines and the polarizability parallel $\alpha_{\mathrm{el}}^{\|}$becomes predominant. Since the frequency dependence of the polarizabilities is different, i.e., the parallel component $\alpha_{\mathrm{el}}^{\|}$can follow the stimulating electric field up to higher frequencies, the maximum of the measured dispersion shifts to higher frequencies.

5.3. $q$-DEPENDENCE. - In the first part of this section we examine the behaviour of systems without intermolecular correlations. For this purpose samples with low particle concentrations $\left(c<c^{*}\right)$ have been prepared, screening the interparticle interaction by adding small amounts of $\mathrm{NaCl}$ to the suspension $\left(\sim 10^{-5}\right.$ molar). If intermolecular interferences can be neglected $(S(q) \approx 1)$ the measured intensity is proportional to the particle form-factor (Eq. (1)). Figure 5 shows the normalized intensity and the theoretical form-factor without and with an external electric field as a function of the magnitude of the scattering vector $q$ for a sample with a particle concentration of $0.5 c^{*}$. The conductivity of the suspension was $5 \mu \mathrm{S} / \mathrm{cm}$. The measured intensity $I_{0}(q)$ in the absence of the field coincides with the theoretical isotropic form-factor $P_{0}(q)$ of equation (6). Slight deviations at small scattering angles are due to a small amount of aggregated fd-virus particles enhancing the forward scattering. The field-free case is compared with the data after a field of $400 \mathrm{~V} / \mathrm{cm}$ and a frequency of $3 \mathrm{kHz}$ has been applied; the measured intensity is normalized by the same constant as in the field free case.

The inset of Figure 5 shows the linear part of the saturation curve $\Delta I\left(E^{2}\right) / I_{0}$ from which the electric anisotropy of a rod $\Delta \alpha_{\mathrm{el}}$ is determined (Eq. (10)). For $E=400 \mathrm{~V} / \mathrm{cm}$ the measured value of $\Delta \alpha_{e l}=6.1 \times 10^{-30} \mathrm{Fm}^{2}$ corresponds to $\gamma=1.2$. A numerical calculation of the formfactor $P(q, \gamma)$ and $\Delta I(q, \gamma) / I_{0}(q)$ for $(\gamma=1.2$ (Eq. (9)) fits the experimental data (see Fig. 5). For higher scattering angles, where the scattered intensity is not sensitive to aggregates, the 


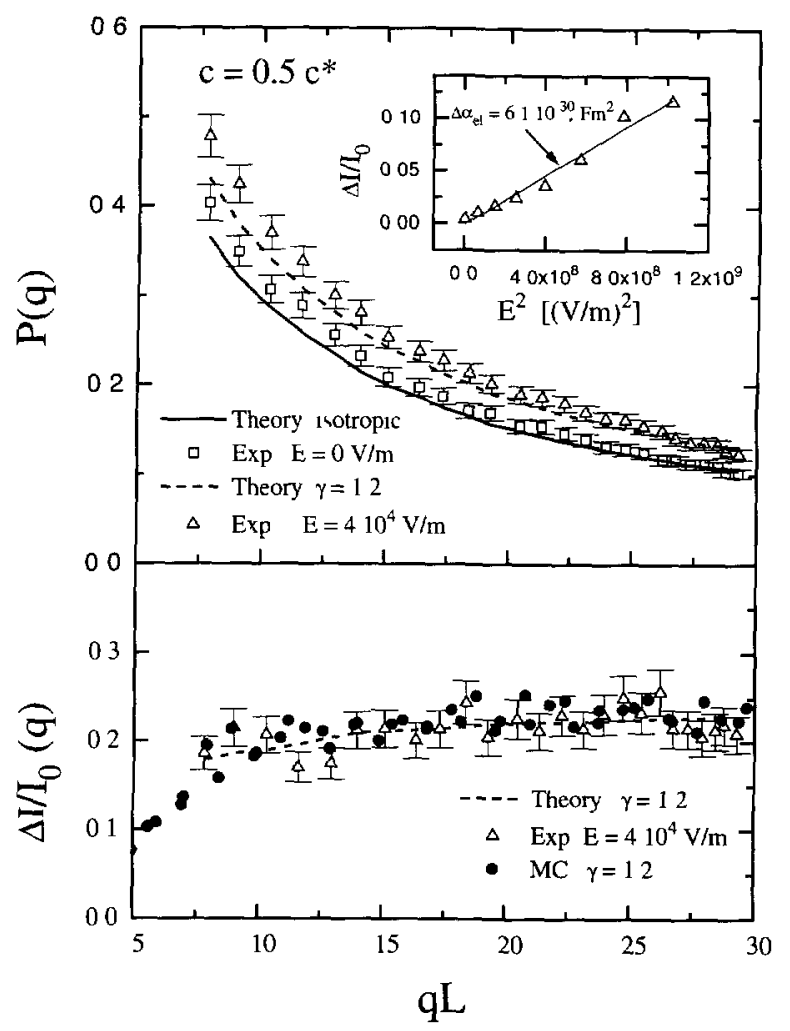

Fig. 5. - The $q$-dependent $\Delta I(q, E) / I_{0}$ and form-factor $P(q, E)$ of a fd-virus suspension with a concentration of $0.5 c^{*}$ at minimal ionic strength. The measured form-factors (open symbols) without/with an external electric field are compared to the theoretical form-factors according to equations $(5) /(9)$ (lines). Additionally, $\Delta I / I_{0}$ and the corresponding $M C$ simulation for $\gamma=1.2$ (solid symbols) are plotted. The experimentally determined electric anisotropy $\Delta \alpha_{\mathrm{el}}=6.1 \times 10^{-30} \mathrm{Fm}^{2}$ (inset) corresponds to $\gamma=1.2$ used for the numerical calculations and the MC simulation.

experimental results are in good agreement with the theory of Stoimenova within experimental error $(<5 \%)$. In addition, these results are confirmed by MC simulations performed for $c=0,5 c^{*}$ and a salt concentration of $10^{-5}$ molar (Fig. 5). It can be concluded that at low particle concentrations the interaction of a single rod with an external electric field described by the coupling parameter $\gamma$ is an appropriate model (Eq. (8)).

Interparticle correlations give rise to a pronounced peak in the static structure factor. Therefore numerical results of the theory derived by Stoimenova (Eq. (9)) are only applicable in the $q$-range where interparticle interferences can be neglected, i.e., $S(q) \approx 1$. This assumption is fulfilled at higher scattering angles depending on the particle concentration and the ionc strength of the suspension. Figure 6 shows experimental, theoretical and $\mathrm{MC}$ simulation results of $\Delta I / I_{0}$ for several electric field strengths as a function of the magnitude of the scattering vector $q$ for a sample with a particle concentration of $c^{*}$ at minimal ionic strength. The external field frequency was $10 \mathrm{kHz}$. The liquid-like character of the isotropic sample $(E=0)$ follows from the observation of a considerable peak (relative peak height $\sim 2$ ) at $q_{\max } L \approx 7$ in the static structure factor, which is not included in the figure. A numerical calculation of $\Delta I(q, \gamma) / I_{0}(q)$ (Eq. (9)) fits the experimental data for all electric field strengths at higher 


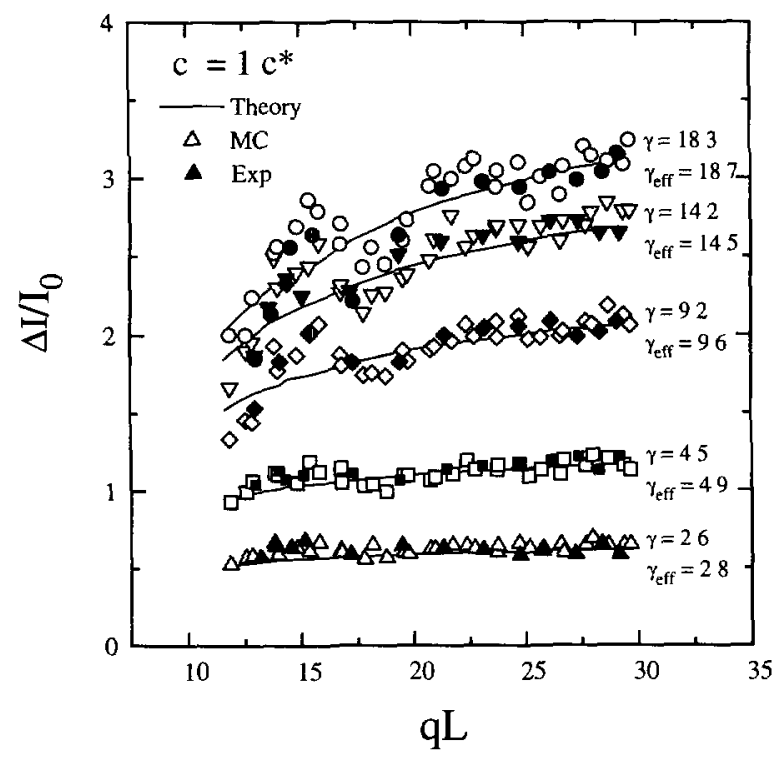

Fig. 6. - The $q$-dependent $\Delta I(q, E) / I_{0}$ of a fd-virus suspension with a particle concentration of $c^{*}$ at minimal ıonic strength for several electric field strengths (solid symbols). The electric field frequency is fixed to $10 \mathrm{kHz}$. Numerical calculations (solid lines) according to equation (9) fit the experimental data yrelding the effective field parameter $\gamma_{\text {eff }}$ The corresponding MC results (open symbols) are performed using the bare field parameter $\gamma$.

$q$-values if $\gamma$ is considered as a fitting parameter denoted by $\gamma_{\text {eff }}$. This quantity parameterizes the effects of interparticle interactions by forcing the effects of the external field on interacting particles to have the same functional form as for a single particle, expressed by the one-particle potential $U_{\text {orient }}$ of equation (8). In the low $q$-range deviations between the measured intensity change $\Delta I(q) / I_{0}$ and the calculations are due to intermolecular interferences $(S(q, \gamma) \neq 1)$.

To examine the influence of the interparticle electrostatic repulsion on the particle alignment in an external electric field we performed MC simulation for several values of $\gamma$ at minimal ionic strength. The $\mathrm{MC}$ results for $\Delta I / I_{0}$ are in good agreement with the experimental data (Fig. 6) if one chooses a $\gamma$-value for the $\mathrm{MC}$ simulations which is in general smaller than the experimentally determined $\gamma_{\text {eff }}$.

The influence of the intermolecular interaction on the orientation distribution functions $f(\Theta)$ will clarify this result. In Figures $7 \mathrm{a}$ and $7 \mathrm{~b}$ the orientation distribution function provided by $\mathrm{MC}$ simulations are compared to Boltzmann orientation distribution functions (Eq. (7)) using both, $\gamma$ the input parameter of the MC simulation and an effective field parameter $\gamma_{\text {eff }}$ in the expression of the orientational energy $U_{\text {orient }}$ (Eq. (8)). $\gamma_{\text {eff }}$ was determined from MC configurations by fitting the Boltzmann orientation distribution to the MC results. For all examined samples we found: $\gamma \leq \gamma_{\text {eff }}$. The difference between the MC value of $\gamma$ and $\gamma_{\text {eff }}$ is less than $5 \%$ for $c<c^{*}$ and increases up to $30 \%$ with increasing particle concentration for a sample with $c=3.6 c^{*}$.

These facts reveal that the interparticle interaction enhances the alignment of the suspended rods in an external electric field. This can be interpreted in terms of a molecular field additional to the external field. With increasing particle concentration the anisotropy of the macromolecules and the anisotropy of their electrostatic potential become more important [18] and 


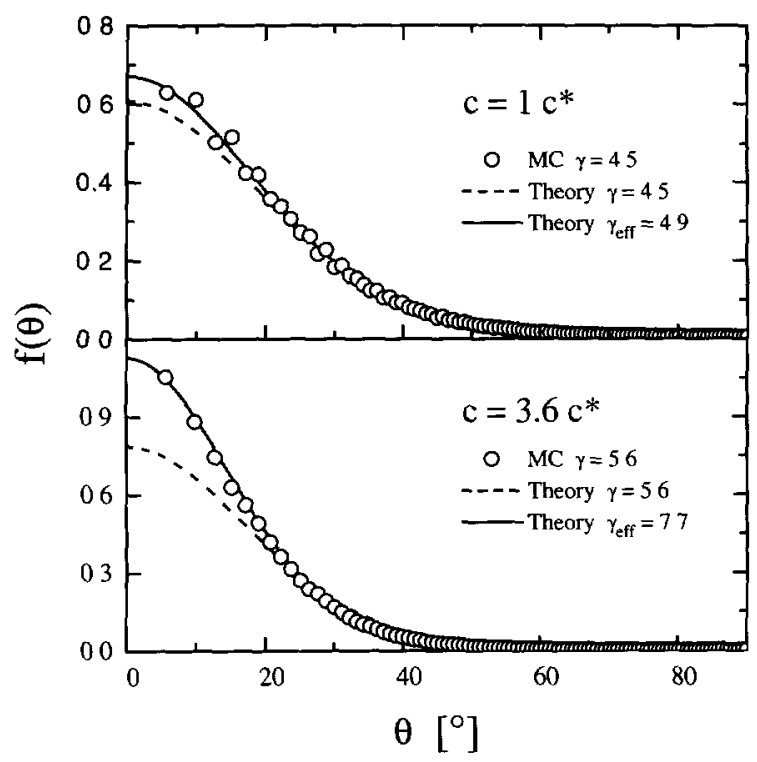

Fig 7 - The orientation distribution $f(\theta)$ from MC simulations (open symbols) performed with the bare field parameter $\gamma$ for a particle concentration of $c=1 c^{*}$ and $c=3.6 c^{*}$. Numerical calculations of the Boltzmann orientation distribution function according to equation (7) for the bare field parameter $\gamma$ (dashed lines) respectively the effective field parameter $\gamma_{\text {eff }}$ (solid lines) are compared to the MC results.

enhance the molecular field, i.e., the difference between $\gamma$ and $\gamma_{\text {eff }}$. This difference vanishes for small particle concentration where the anisotropic part of the electrostatic potential is small compared to the isotropic part which has no influence on the particle orientations. For very high electric fields the influence of the external field is predominant and the difference is less than for smaller electric fields. This qualitative explanation is in good accordance with our investigations. We conclude that the influence of the interparticle interaction on the orientation distribution function can be described by an effective one-particle orientation potential $U_{\text {orient }}$ where the bare field parameter $\gamma$ is replaced by its effective value $\gamma_{\text {eff }}$. It is this parameter which yields the correct Boltzmann form of the orientation distribution function.

In the following section the influence of the electric field strength on the polarizability of the macromolecules will be examined. At low degree of particle orientation $\Delta \alpha_{\text {el }}$ is proportional to the measured EOE and can be determined by means of equation (10). This method is restricted to the Kerr regime. At higher electric fields a comparison between the experimental data $\Delta I / I_{0}$ and MC simulations provides the bare field parameter $\gamma$ respectively the electric anisotropy of a single particle $\Delta \alpha_{\mathrm{el}}$. Figure 8 shows the dependence of $\Delta \alpha_{\mathrm{el}}(E)$ on the external electric field strength. Here, $\gamma(E)$ is plotted as a function of $E^{2}$ for the sample of Figure 6 with a particle concentration of $c^{*}$ at minimal ionic strength. At low degree of particle orientation, $\gamma$ is proportional to $E^{2}$ and $\Delta \alpha_{\mathrm{el}}=$ const. and corresponds to the value $\Delta \alpha_{\mathrm{el}}=1.5 \times 10^{-29} \mathrm{Fm}^{2}$ determined in the Kerr-regime (Eq. (10)). For $\gamma>1$ the electric anisotropy $\Delta \alpha_{\text {el }}(E)$ decreases with increasing electric field strength. At electric field strengths above $2.5 \times 10^{5} \mathrm{~V} / \mathrm{m}, \gamma$ remains constant corresponding to a decrease of $\Delta \alpha_{\mathrm{el}}(E) \propto E^{-2}$. Nevertheless the maximum experimental value of $\gamma_{\mathrm{eff}}\left(E \geq 2.5 \times 10^{-5} \mathrm{~V} / \mathrm{m}\right) \approx 23$ indicates a strong particle alignment along the electric field according to the orientational order parameter $\phi(\gamma=23) \approx 0.93(\mathrm{Eq}$. (11)). 


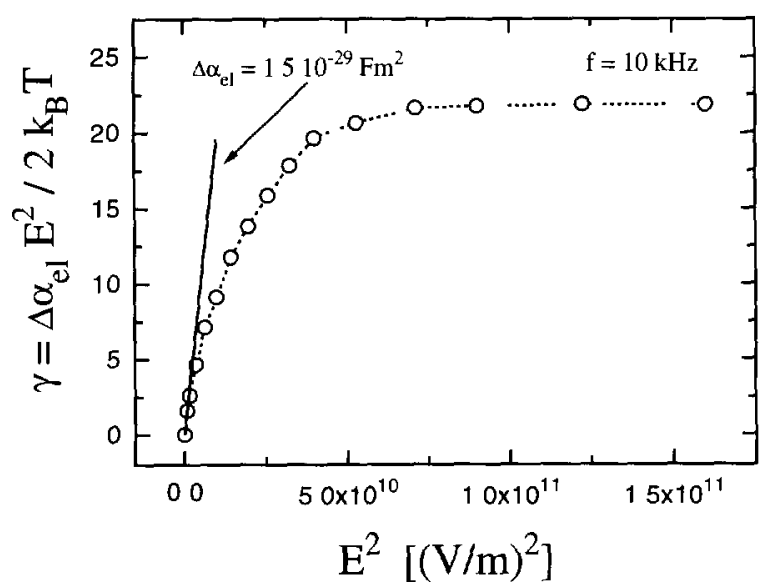

Fig. 8. - The electric field strength dependence of $\gamma=\Delta \alpha_{\mathrm{el}} E^{2} / 2 k_{\mathrm{B}} T$ (open symbols) for a fd-virus suspension with a particle concentration of $1 c^{*}$ at minimal ionic strength and a field frequency of $10 \mathrm{kHz}$. The solid line corresponds to $\Delta \alpha_{\mathrm{el}}=1.5 \times 10^{-29} \mathrm{Fm}^{2}$ experimentally determined in the Kerr regime for this sample (Eq. (10)).

A decrease of $\Delta \alpha_{\mathrm{el}}(E)$ with increasing field strengths is theoretically explained by several authors $[43,56,57]$ by a stripping of the counterions away from the electrostatic influence of the central rod. Fixman and Jagannathan [56] have shown that at moderate electric field strengths of several $100 \mathrm{~V} / \mathrm{cm}$ the induced dipole is no longer proportional to the electric field strength, and the counterion atmosphere starts to be stripped away. In high electric fields Rau and Charney [43] have predicted a decrease of the polarizability of the diffuse part of the counterion atmosphere proportional to $E^{-2}$. This leads to $\gamma \approx$ const. and therefore an incomplete orientation of the suspended particles, even at very high field strengths. These theoretical considerations are confirmed by MC simulations for rod-like DNA particles performed by Yoshida et al. [58]. Our experimental results fully support these considerations.

In the preceding sections we have discussed the influence of rod alignment on light scattering at large scattering angles where interparticle correlations can be neglected. Now we discuss the effects of the electric field for the whole $q$-regime. For that purpose, we compare the light scattering results of a sample with a particle concentration of $3.6 c^{*}$ at minimal ionic strength with MC simulation data. Previous work on isotropic fd and TMV systems [18, 19] has demonstrated that it is possible to describe static light scattering properties of these suspensions using the interaction potential of equation (13). Here, we examine if this holds for a system with an orienting electric field.

In Figure 9 snapshots of thermalized MC configurations for several electric field strengths are displayed. They represent projections of the rod axis on the $x y$ - and $x z$-planes. The alignment due to the external electric field (in the $\mathbf{e}_{z}$-direction) is clearly demonstrated. The strong particle alignment for $\gamma=19.6$ is in good agreement with the orientational order parameter $\Phi(\gamma \approx 20) \approx 0.92$, which is close to 1 .

The resulting structure factor for these systems is shown in Figure 10 together with experimental electric field light scattering data. The experimental structure factors $S\left(q, \gamma_{\text {eff }}\right)$ were obtained by determining $\gamma_{\text {eff }}$ and a numerical calculation of the form-factor $P\left(q, \gamma_{\text {eff }}\right)$ according to equation (9). Then the normalized scattered intensities $I(q, E)$ are divided by the corresponding form-factor $P\left(q, \gamma_{\text {eff }}\right)$. For the $\mathrm{MC}$ simulations a field variable $\gamma$ was chosen such that 

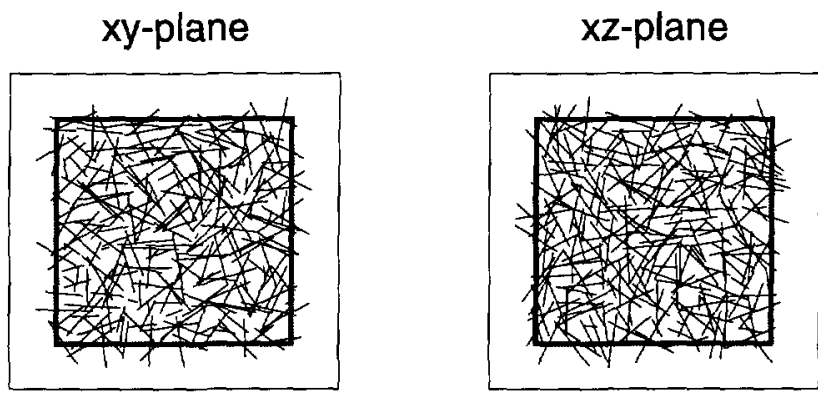

\section{isotropıc}
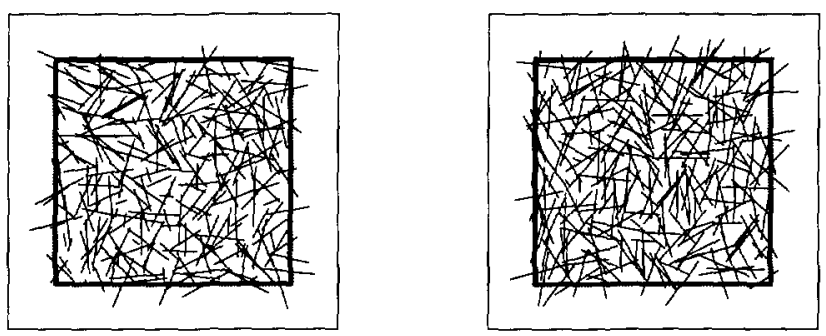

$\gamma=1.0$

$\Phi(\gamma)=0.19$
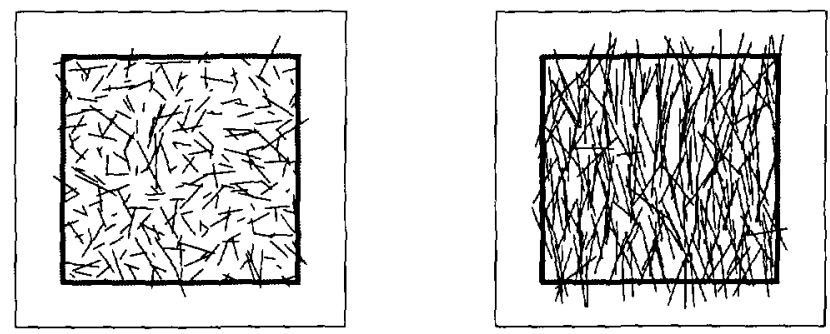

\section{$\gamma=5.6$ \\ $\Phi(\gamma)=0.77$}
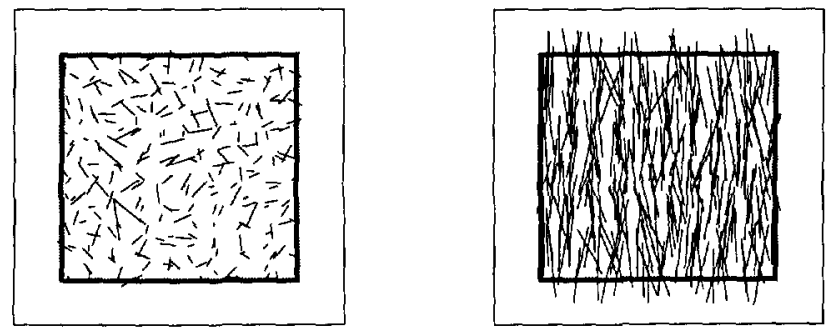

$$
\begin{aligned}
& \gamma=19.6 \\
& \Phi(\gamma)=0.93
\end{aligned}
$$

Fig. 9. - Snapshots of thermalized MC configuratıons for several electric field strengths for a particle concentration of $3.6 c^{*}$ They represent projections on the rods axes on the $x y$ - and $x z$-plane. The external field is parallel to $\mathbf{e}_{z}$. The degree of particle alignment is indicated by the orientational order parameter $\Phi(\gamma)$. 


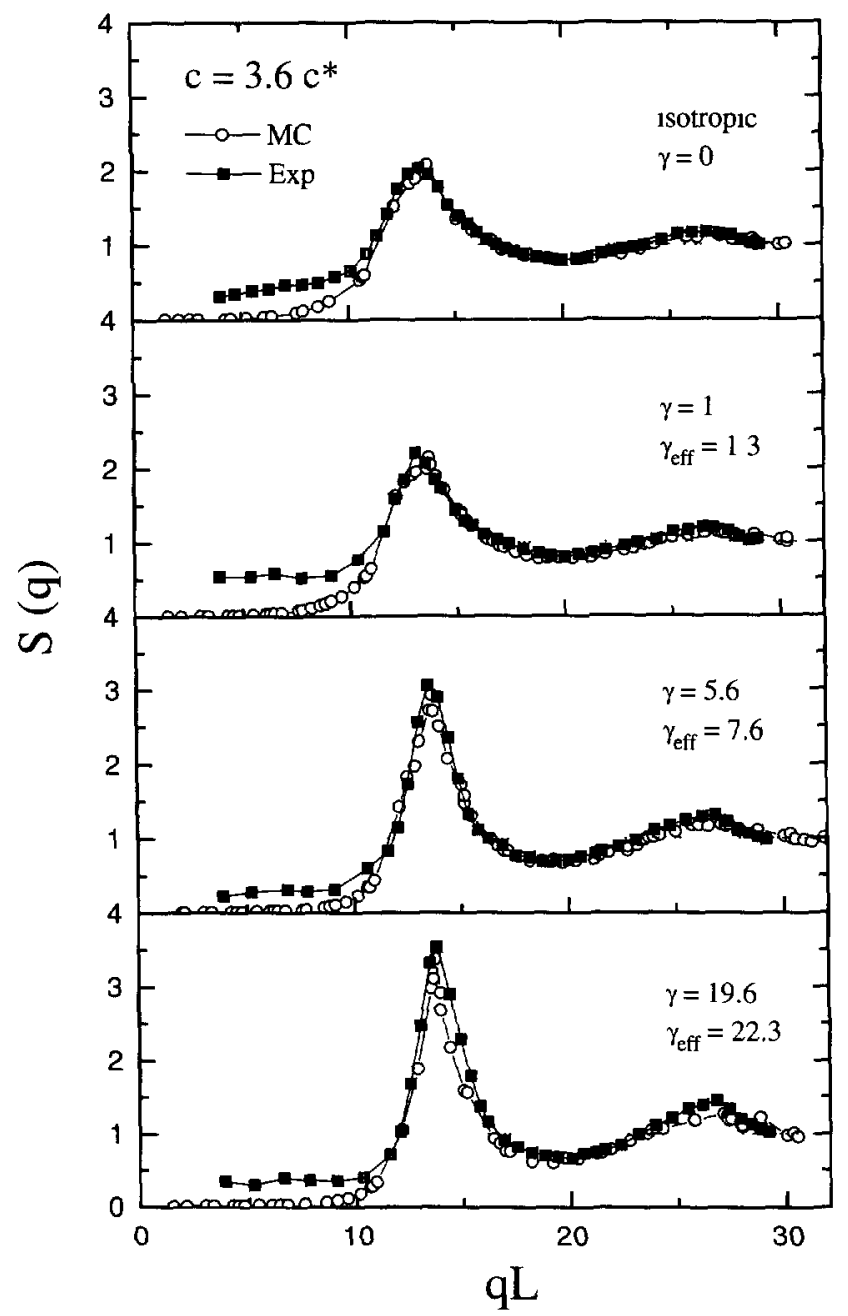

Fig. 10. - The static structure factor $S(q, E)$ of a fd-virus suspension of $3.6 c^{*}$ at minimal ionıc strength for several electric field strengths (solid symbols) The electric field frequency is fixed to 10 $\mathrm{kHz}$. The MC simulations (open symbols) match the experimental data for a bare field parameter $\gamma$ whereas the experimental results provide the effective field parameter $\gamma_{\mathbf{e f f}}$.

the resulting value for $\gamma_{\text {eff }}$, determined as described previously, matches the experimental value. Besides the effective $\mathrm{MC}$ charge $\mathrm{Q}$, which was set to $420 \mathrm{e}^{-}$in order to get good agreement between $\mathrm{MC}$ and light scattering results in the field free case, there is no additional adjustable parameter in this procedure. Nevertheless the agreement remains good for all field strengths. For very low values of $q$, there is a notable discrepancy between experimental and MC results. This can be explained by the presence of a small amount of aggregated virus particles. These large aggregates scatter light predominantly in the forward direction, therefore experimental $S\left(q, \gamma_{\mathrm{eff}}\right)$ is apparently increased in the low $q$-regime.

The effect of the external electric field is a structure peak which is higher as in the isotropic case indicating an increased order in the system. For a high degree of particle alignment the 
structure factor represents mainly a measure of the order of the rods centers of mass, since in the case of complete particle alignment along the electric field the expression for $S(\mathbf{q})$ (Eq. (12)) is simplified to:

$$
S(\mathbf{q}, \gamma) \stackrel{\gamma \rightarrow \infty}{\longrightarrow} S_{\mathrm{CC}}(\mathbf{q})=1+\frac{1}{N}\left\langle\sum_{\imath \neq j}^{N} \mathrm{e}^{\imath \mathbf{q}\left(\mathbf{R}_{z}-\mathbf{R}_{2}\right)}\right\rangle
$$

$S_{\mathrm{CC}}(\mathbf{q})$ describes the statıc structure factor of the centers of mass for totally aligned rods. This expression for $S(\mathbf{q})$ is identical to the case of spherical symmetric particles. A quantitative measure of this order is the pair correlation function of the macromolecules. Results for this quantity from MC simulations are plotted in Figure 11. The averaged center-center pair correlation is defined by:

$$
g_{\mathrm{CC}}(R):=\left\langle g\left(\mathbf{R}, \mathbf{u}_{\imath}, \mathbf{u}_{\jmath}\right)\right\rangle_{\hat{\mathbf{R}}, \mathbf{u}_{i}, \mathbf{u}_{\jmath}}, \quad \hat{\mathbf{R}}=\frac{\mathbf{R}}{|\mathbf{R}|} .
$$

$g_{\mathrm{CC}}$ shows little structure in the absence of an external field for the optically isotropic sample. An increase of the field strength enhances the alignment of the particles and also the order in the system. This effect becomes even more pronounced, if one looks at correlations perpendicular to the field direction, i.e., for pairs of molecules with mutual distance vector $\mathbf{R} \perp \mathbf{E}$. Light scattering with a scattering vector $\mathbf{q} \perp \mathbf{E}$ is specifically sensitive for this type of correlations.

The structural change due to an external electric field can roughly be explained in the following way: for optically isotropic suspensions, a strong ordering of the macromolecules centers of mass would require highly correlated axes. This conformation has a small orientational entropy and is therefore thermodynamically unfavourable compared to a conformation with less order. In the case of an high external electric field $(\gamma>1)$ the orientational entropy is small with

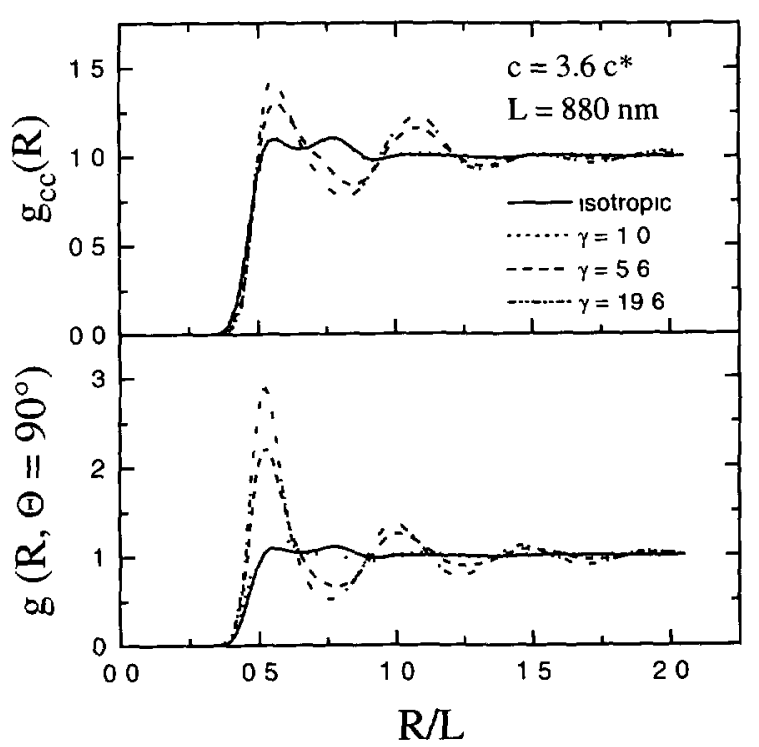

Fig. 11. - The averaged center-center pair correlation function $g_{\mathrm{cc}}(R)$ for several $\gamma$-values (upper part). The pair correlation function perpendicular to the external field direction $g\left(R, \theta=90^{\circ}\right)$ is plotted in the lower diagram. The solid lines denote the isotropic case for $\gamma=0$. 
respect to orientation energy and the macromolecules can order more easily in configurations which reduce the intermolecular electrostatic repulsion.

5.4. Low-Field Anomaly. - Figure 4 shows that for samples with high particle concentrations and minimal ionic strength $\Delta I / I_{0}$ becomes negative in the frequency range below $500 \mathrm{~Hz}$. An anomalous behaviour of the EOE is well known for strongly interacting systems but not yet understood [12,13,45-48]. The low-field anomaly $\left(\Delta I / I_{0}<0\right)$ has a magnitude of a few percent and can be distinguished from $I_{0}(q)$ if the statistics of a measurement is sufficiently good. Experimentally we find $\Delta I / I_{0}<0$ clearly for samples with fd-particle concentrations of at least $2.5 c^{*}$ and minimal ionic strength. In electric birefringence measurements, which are more sensitive to particle orientation, we found a negative birefrungence signal for $\mathrm{fd}$-particle concentrations of about $c^{*}[12,13]$. In Figure 12 the dependence of the low field anomaly is plotted as a function of the external electric field strength for several particle concentrations at

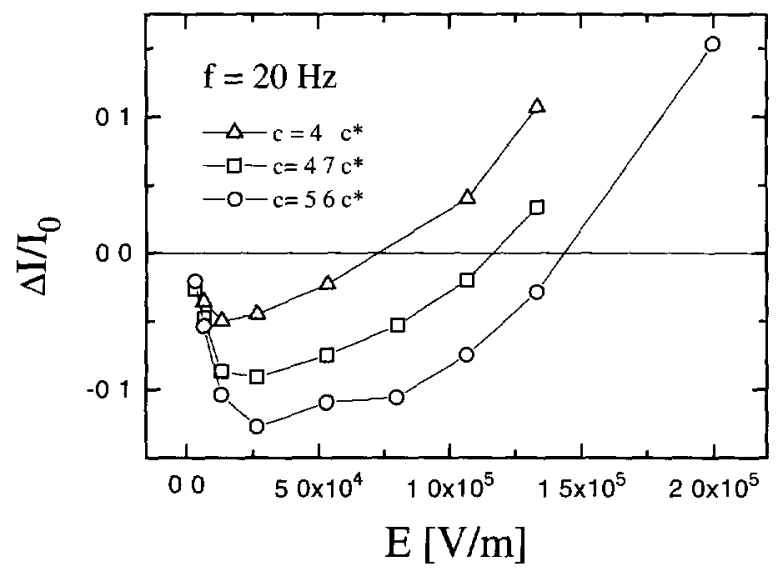

Fig. 12. - The electric field strength dependent $\Delta I / I_{0}$ of fd-virus suspensions at minımal conductivities and a field frequency of $20 \mathrm{~Hz}$. The scattering angle is fixed to $140^{\circ}$

a fixed frequency of $20 \mathrm{~Hz}$. The anomalous behaviour changes to positive or normal behaviour with increasing electric field strength. The negative EOE disappears also if the intermolecular interaction is screened [13]. The anomaly is therefore a collective effect of strongly interacting rods. According to the theoretical considerations a decrease of the scattered light intensity corresponds to a particle orientation where the rods symmetry axis prefers to align perpendicular to the applied field (see Fig. 1). The largest anomaly with a value of $\Delta I / I_{0} \approx-0,13$ $\left(\vartheta=140^{\circ}\right)$ is found for the fd-virus concentration of $5.6 c^{*}$. If all rods are aligned in the observation plane $\left(\mathbf{u} \perp \mathbf{e}_{z}\right)$ the theoretically expected change of the scattered light intensity amounts to $\Delta I / I_{0} \approx-0,4\left(\vartheta=140^{\circ}\right)$, as follows from equation (9). The measured anomaly effect is significantly below this saturation value.

Particle alignment perpendicular to the electric field can only be explained if the polarizability perpendicular to a rod axis $\alpha_{\mathrm{el}}^{\perp}$ is significant. Fixman and Jagannathan $[49,52]$ showed that for rod-like particles at very low ionic strength the magnitude of $\alpha_{\mathrm{el}}^{\perp}$ is comparable to that of $\alpha_{\mathrm{el}}^{\|}$. Further, for extended counterion clouds the electrophoretic movement of the particles relative to the solvent causes an additional convective polarization which can dominate the electric polarizability of the counterion cloud. 
Different mechanism to understand the low field anomaly of strongly interacting rod-like polyelectrolytes have been suggested by Hoffmann et al. [47,48], Cates [53] and Odijk [54]. A survey and discussion of these ideas was given by Kramer $[13,55]$. From our MC results we infer that as long as the interparticle potential is correctly described by the DLVO expression (Eq. (13)), the particles do not align perpendicular to the field in the case of $\Delta \alpha_{\mathrm{el}}>0$. We assume that the anomaly has to be attributed to the collective behaviour of the system consisting of the macromolecules and their strongly overlapping diffuse counterion clouds. However, a precise theoretical treatment of the low field anomaly is beyond the scope of this work.

\section{Conclusions}

Different aspects due to the particle alignment in an external electric field of rod-like fdvirus macromolecules suspended in aqueous suspensions at very low ionic strengths have been examined by electric field light scattering and MC simulations. The MC simulations were based on the model potential describing the interparticle electrostatic repulsion (DLVO), as well as the coupling of a single particle to the external electric field via the coupling parameter $\gamma$. The experimental results could be reproduced by the MC simulations which provide additional insights in the microstructure of the suspension.

The electric field light scattering investigations confirm and extend our previous electric birefringence experiments $[12,13]$ concerning the dispersion and field strength behaviour of fdvirus suspensions. At low ionic strengths the fd-virus particles are oriented by an induced dipole originating predominantly from a polarization of the diffuse part of the counterion cloud in an external electric field. Furthermore, to our knowledge, we could measure quantitatively the dependence of the electric anisotropy $\Delta \alpha_{\mathrm{el}}$ of a macromolecule as a function of the electric field strength for the first time. We found a decrease of the electric anisotropy $\Delta \alpha_{\mathrm{el}}$ with increasing field strength. This is interpreted as a stripping of the counterions away from the electrostatic influence of the central rod leading to a decrease of its polarizability. In high electric fields we found experimentally: $\Delta \alpha_{\mathrm{el}} \propto E^{-2}$, i.e., $\gamma_{\max } \approx$ const. Nevertheless, indicated by the orientational order parameter $\Phi\left(\gamma_{\max }\right) \approx 0.93$, a high degree of particle alignment along the electric field can be attained. These results agree well with theoretical predications considering the diffuse counterion atmosphere in an external electric field $[43,56,58]$.

For systems with screened interparticle interaction the experimental results as well as the $\mathrm{MC}$ simulations correspond to the theoretical treatment of the relative change of the scattered light $\Delta I / I_{0}$ derived by Stoimenova for non-interacting rod-like particles. Deviations occur in the range where interparticle interferences are significant $(S(q) \neq 1)$ and for strongly interacting systems at particle concentrations $c \geq c^{*}$. For such systems we demonstrated that the interparticle electrostatic repulsion increases the particle alignment in an external electric field considerably. Furthermore the coupling of a single macromolecule to the electric field could be described sufficiently with a one-particle potential by replacing the bare field parameter $\gamma$ by an effective $\gamma_{\mathrm{eff}}\left(\gamma \leq \gamma_{\mathrm{eff}}\right)$.

We found that an application of an external electric field enhances the structure in the system considerably. This is indicated by an increase in the peak height of the static structure factor and the center-center pair correlation function with regard to the isotropic case.

Besides increasing the particle alignment in external field, strongly interacting systems above the overlap concentration show anomalous particle orientation perpendicular to the external field at low electric field strengths and field frequencies below $1 \mathrm{kHz}$. We conclude that collective effects of the strongly overlapping counterion clouds are responsible for a significant polarizability perpendicular to the rods axis leading to the negative sign of $\Delta I / I_{0}$.

The complementary methods of transient electric birefringence, electric field light scattering 
and MC simulation give us extensive information about the properties in colloidal suspensions of rod-like polyelectrolytes.

\section{Acknowledgments}

This work was supported by the Deutsche Forschungsgemeinschaft (Grant SFB 306).

\section{References}

[1] Halsey T.C., Scrence 258 (1992) 761.

[2] Block H. and Kelly J.P., J Phys. D: Appl. Phys. 21 (1988) 1661

[3] Kalus J.and Hoffmann H., J. Chem Phys 87 (1987) 714.

[4] Frank H. and Day L.A., Virology 42 (1970) 144.

[5] Newman J., Swinney H L. and Day L A , J. Mol Bıol 116 (1977) 593.

[6] Maeda T and Fujıme S., Macromolec. 18 (1985) 2430.

[7] Beck K and Duenki R.M., J. Struct. Bıol. 105 (1990) 22.

[8] Zimmermann K., Hagedorn H., Heuck C.C., Hindrichsen M. and Ludwig H., J Bıol. Chem. 261 (1986) 1653.

[9] Hoss U., Batzill S., Deggelmann M , Hagenbüchle M., Johner C., Kramer H , Martin C., Overbeck E. and Weber R., Macromolec 27 (1994) 3429.

[10] Hunter R.J., Zeta Potential in Colloid Science Principles and Applications (Academic Press, London, 1981)

[11] Kramer H., Deggelmann M., Graf C., Hagenbuichle M, Johner C. and Weber R., Macromolec. 25 (1992) 4325.

[12] Kramer H., Martin C., Graf C., Hagenbüchle M, Johner C. and Weber R., Prog Coll. Int. Scı. 97 (1994) 40.

[13] Kramer H., Graf C., Hagenbüchle M., Johner C., Martin C., Schwind P. and Weber R., J Phys. II France 4 (1994) 1061

[14] Stoylov S P., Electro-Optics Theory, Techniques, Applicatıons (Academıc Press, London, 1991).

[15] Jennings B R., Molecular Electro-Optıcs, C T O'Konski, Ed (Dekker, New York, 1976).

[16] Stoylov S P. and Stomenova M.V, J Coll. Int Scı 40 (1972) 154

[17] Stoimenova M.V., J. Coll Int. Scr. 53 (1975) 42.

[18] Hagenbüchle M, Weyerıch B., Deggelmann M, Graf C, Krause R, Maıer E., Schulz S., Klein R. and Weber R., Physica A 169 (1990) 29.

[19] Maier E, Krause R., Deggelmann M, Hagenbüchle M and Weber R., Macromolec. 25 (1992) 1125.

[20] Berne J B. and Pecora R., Dynamic Light Scatterıng (John Wley \& Sons, New York, 1976).

[21] Van der Hulst H.C., Light Scattering by Small Particles (Dover, New York, 1981).

[22] Buitenhuis J., Dhont J K.G. and Lekkerkerker H.N W, J. Coll Int Scr 162 (1994) 19.

[23] Stoylov S.P, Adv. Coll. Int. Sci. 3 (1971) 45.

[24] Wippler C., J. Chem. Phys. 53 (1956) 328.

[25] Neugebauer T., Ann. Phys. 42 (1943) 509.

[26] Fredericq E. and Houssier C., Electric Dichroism and Electric Birefringence (Clarendon Press, Oxford, 1973).

[27] O'Konski C.T., Yoshioka K and Orttung W.H., J. Phys Chem. 63 (1959) 1558. 
[28] Dawson H., Proc. London Math. Soc. 291898519.

[29] Gray C.G. and Gubbins K.E , Theory of Molecular Fluids (Clarendon Press, Oxford, 1984).

[30] Canessa E., D'Aguanno B., Weyerıch B. and Klein R., Mol Phys. 73 (1991) 175.

[31] Callol J.M and Weis J.J, J. Chem Phys. 90 (1989) 7403

[32] Schneider J., Hess W and Klein R., J. Phys. A 18 (1985) 1221

[33] Schneider J., Karrer D., Dhont J.K.G. and Klein R., J. Chem Phys. 87 (1987) 3008.

[34] Löwen H., Phys. Rev Lett. 72 (1994) 424.

[35] Löwen H., J. Chem. Phys. 100 (1994) 6738

[36] Marvin D A. and Wachtel E J., Nature 253 (1975) 19.

[37] Uzgiris E.E., Adv Coll. Int. Scr. 14 (1981) 75

[38] Deggelmann M., Palberg T., Hagenbuichle M., Maier E., Krause R, Graf C. and Weber R., J Coll Int. Scr 143 (1991) 318.

[39] Deggelmann M., Graf C., Hagenbuchle M., Hoss U., Johner C., Kramer H., Martin C. and Weber R., $J$ Phys Chem 98 (1994) 364

[40] Martin C, Kramer H.,Johner C., Weyerich B, Biegel J., Deike R, Hagenbüchle M. and Weber R., Macromolec., to appear in the May 1995 issue.

[41] Hogan M., Dattagupta N. and Crothers D.M , Brochemrstry 75 (1978) 195.

[42] Rau D.C and Bloomfield V.A, Bıopolymers 18 (1979) 2783.

[43] Rau D C. and Charney E., Brophys. Chem. 17 (1983) 35.

[44] Rau D.C. and Charney E., Brophys. Chem. 14 (1981) 1.

[45] Lauffer M.A., J. Am. Chem Soc. 61 (1939) 2412.

[46] Asai H. and Watanabe N., Bropolymers 15 (1976) 283

[47] Hoffmann H., Kramer U. and Thurn H., J. Phys. Chem. 94 (1989) 2027.

[48] Kramer U. and Hoffmann H., Macromolec 24 (1991) 256

[49] Fixman M. and Jagannathan S., J Chem. Phys. 75 (1981) 4048.

[50] Oosawa F., Biopolymers 9 (1970) 677.

[51] American Institute of Physics Handbook (New York, 1972).

[52] Fixman M., J. Chem. Phys 75 (1981) 4040.

[53] Cates M E., J. Phys. II France 2 (1992) 1109.

[54] Odijk T., Laser Light Scattering in Biochemistry (London Roy. Soc. Chem., 1992).

[55] Kramer H., Ph.D. Thesis, Konstanz, ISBN 3-89191-681-7 (1993).

[56] Fixman M. and Jagannathan S., Macromolec. 16 (1983) 685.

[57] Altıg J.A., Wesenberg G.E. and Vaughan W E, Brophys Chem 24 (1986) 221.

[58] Yoshıda M., Kikuchi M., Maekawa T and Watanabe H., J. Phys. Chem. 96 (1992) 2365 